03

\title{
Динамические эффекты в магнитной жидкости с микрокаплями концентрированной фазы во вращающемся магнитном поле
}

\author{
(C) Ю.И. Диканский, М.А. Беджанян, А.А. Колесникова, А.Ю. Гора, А.В. Чернышев \\ Северо-Кавказский федеральный университет, \\ 355009 Ставрополь, Россия \\ I e-mail: anastasiya.kolesnikova.93@mail.ru
}

(Поступило в Редакцию 18 июня 2018 г. В окончательной редакции 21 сентября 2018 г.)

Исследована динамика формы микрокапель высококонцентрированного магнитного коллоида, образующихся в результате фазового расслоения магнитной жидкости. Показано значительное влияние весьма слабых магнитных полей, соизмеримых с земным полем, на геометрию и поведение микрокапель. Рассмотрены различные конфигурации микрокапель во вращающемся магнитном поле. Показано возникновение вращающего момента, действующего на макроскопическую каплю магнитной жидкости во вращающемся поле в результате внутренних вращений содержащихся в ней микрокапель концентрированной фазы. Измерена частота макроскопического вращения поверхности капли в зависимости от частоты и напряженности внешнего магнитного поля.

DOI: 10.21883/JTF.2019.03.47171.242-18

\section{Введение}

Вращение ограниченных объемов жидких масс в различных масштабах представляет традиционный объект исследования, сохраняя свою актуальность и в настоящее время. Новые возможности в подобного рода исследованиях появились благодаря применению магнитных жидкостей, которыми можно управлять при помощи внешних магнитных полей. Магнитные жидкости представляют собой ультрадисперсный коллоидный раствор ферро- или ферримагнитных наночастиц в несущей жидкости. Был выполнен ряд исследований динамки капель магнитных жидкостей во внешних стационарных, а также вращающихся магнитных полях [1-5]. Развитие новых направлений применения магнитных жидкостей в микофлюидных технологиях [6] побудило к исследованию закономерностей поведения микрокапель магнитных жидкостей, подверженных действию вращающегося поля, при различных внешних условиях. Так, в работах [7-9] исследуется динамика формы микрокапель магнитных жидкостей, диспергированных в немагнитной среде, обладающей малым межфазным натяжением на границе с магнитной жидкостью, а также немагнитных микрокапель, помещенных в магнитную жидкость, при воздействии стационарных и вращающихся магнитных полей. Подобные системы были также получены путем фазового расслоения водных магнитных жидкостей, стабилизированных электростатически [10-13]. В последнем случае при добавлении избыточного электролита в среде образовывались высококонцентрированные микрокапли магнитного коллоида.

Динамика отдельных микрокапель во вращающемся магнитном поле должна приводить к макроскопическим гидродинамическим эффектам в среде, представляющей собой систему таких микрокапель. Вращающий момент, действующий на отдельные капли, за счет вязких сил должен передаваться среде в целом. Подобные эффекты внутренних вращений исследовались ранее в отношении исходных (однородных) магнитных коллоидов [14-17], где они были относительно слабо выражены, а также в отношении магнитных коллоидов, содержащих квазитвердые агрегаты дисперсных частиц [18], и в отношении магнитных эмульсий с высоким межфазным натяжением на границе дисперсной фазы и дисперсионной среды [19], где ротационный эффект был более ярко выражен.

В настоящей работе исследуется поведение отдельных микрокапель с высоким содержанием магнитного вещества, возникающих при фазовом расслоении магнитного коллоида и обладающих более ярко выраженными магнитными свойствами, чем это имело место в предыдущих исследованиях. Рассматривается их поведение в стационарном и вращающемся магнитных полях, а также изучается возникновение вращающего момента, действующего на макроскопическую каплю магнитного коллоида с микрокаплями концентрированной фазы.

\section{Динамика отдельных микрокапель концентрированной фазы}

В экспериментах применялась магнитная жидкость, представляющая собой дисперсию наночастиц магнетита в вакуумном масле с олеиновой кислотой в качестве стабилизатора. При добавлении небольшого избытка стабилизатора наблюдалось фазовое расслоение системы и образование конденсированной фазы в виде высококонцентрированных микрокапель коллоида. Производилось изучение динамики данных микрокапель путем наблюдения в оптический микроскоп. Образец при этом располагался в однородном магнитном поле, создаваемом катушками Гельмгольца. Для создания вращающегося 


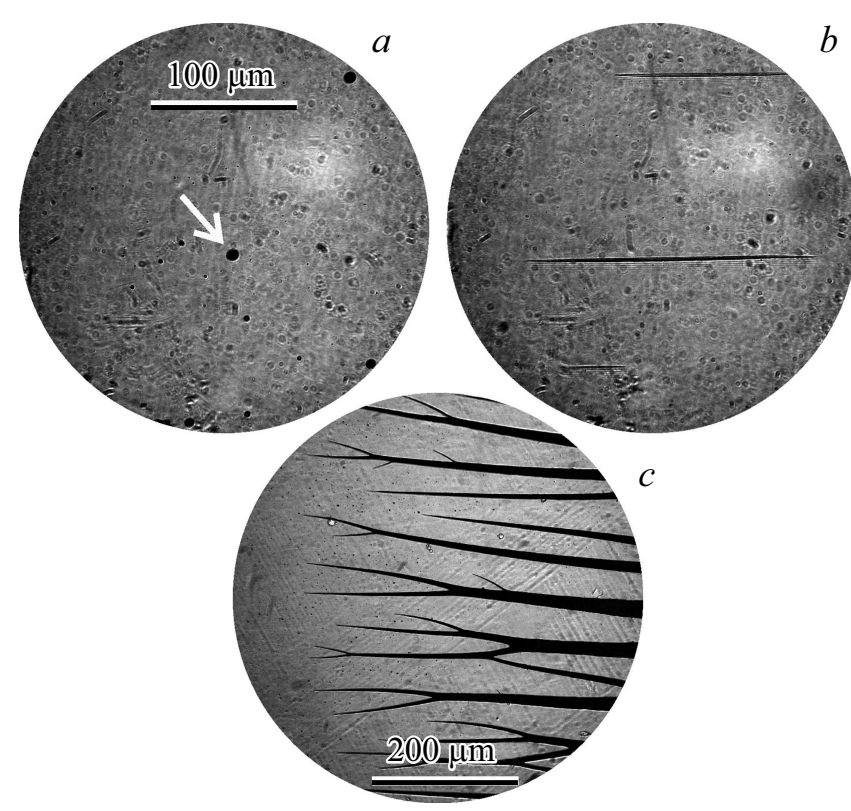

Рис. 1. Изображение микрокапель концентрированной фазы: $a-$ в отсутствии магнитного поля, $b-$ под действием приложенного стационарного магнитного поля напряженностью $140 \mathrm{~A} / \mathrm{m}, c$ - большие микрокапли, деформированные магнитным полем Земли.

магнитного поля применялись две пары скрещенных катушек Гельмгольца, на которые подавалось напряжение со сдвигом фаз $\pi / 2$. При действии стационарного магнитного поля, направленного горизонтально вдоль плоскости образца, наблюдалась деформация капель, при которой они вытягивались вдоль направления поля. При этом деформация носила пороговый характер, а именно при достижении некоторой критической величины магнитного поля наблюдалось значительное скачкообразное удлинение капли (рис. 1). Подобное поведение капель магнитной жидкости наблюдалось и ранее. Отличием настоящего случая является величина скачка при деформации капли и напряженность поля, при котором данный скачок происходит. Вследствие существенно более высоких значений магнитных характеристик полученных микрокапель их деформация имеет место в весьма слабых магнитных полях, лишь не на много отличающихся от земного, а наблюдаемое при этом удлинение заметно превосходит имевшее место в предшествующих исследованиях.

В качестве примера на рис. 2 приведена экспериментальная зависимость отношения полуосей магнитной микрокапли от величины напряженности магнитного поля. Как видно из рисунка, критическая напряженность здесь лишь в несколько раза превосходит напряженность земного поля. При этом критическая напряженность зависит также от размера микрокапли, поскольку процесс ее деформации определяется балансом магнитных и капиллярных сил. Вследствие этого критическое поле снижается для крупных капель и повышается для мелких (рис. 2). В результате, как было обнаружено в экспериментах, достаточно крупные микрокапли оказываются деформированными под действием лишь земного поля (рис. 1). Отметим, что при измерении зависимостей, представленных на рис. 2, магнитное поле катушек Гельмгольца устанавливалось параллельно горизонтальной составляющей магнитного поля Земли. Следует также заметить, что деформация капель магнитной жидкости в постоянном поле может носить гистерезисный характер [10], заключающийся в различии формы капли при увеличении внешнего поля и его последующем уменьшении. Подобное явление имело место и в рассматриваемом в настоящей работе случае, однако его количественное исследование затруднено влиянием магнитного поля Земли, и поэтому здесь не приводится.

Во вращающемся в горизонтальной плоскости, совпадающей с плоскостью наблюдений, магнитном поле наблюдались различные режимы деформации микрокапель. Было выделено три таких режима. При низких значениях частоты вращающегося поля (порядка нескольких $\mathrm{Hz}$ ) и при малых величинах напряженности поля $(<1 \mathrm{kA} / \mathrm{m})$ капли совершали вращение вслед за направлением поля, сохраняя вытянутую форму. При увеличении частоты вращения магнитного поля, а также при возрастании его напряженности, наблюдалось образование различных переходных форм капель (рис. 3). При дальнейшем росте напряженности магнитного поля $(>5 \mathrm{kA} / \mathrm{m})$ практически независимо от его частоты образовывались конфигурации микрокапель в виде сплюснутых в плоскости поля эллипсоидов вращения (тонких дисков) (рис. 3). Отметим, что в случае близкого расположения

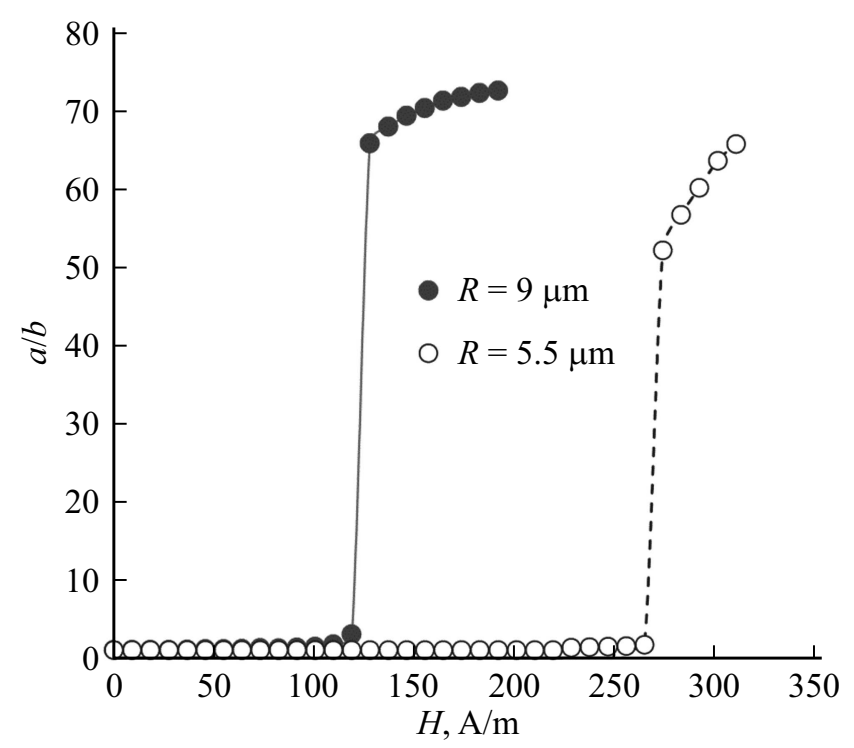

Рис. 2. Экспериментальные зависимости отношения полуосей ( $a$ - большая полуось, $b$ - малая полуось) деформированных микрокапель концентрированной фазы от напряженности приложенного магнитного поля $H$ для капель различного размера (указан радиус капли в отсутствии поля). 




Рис. 3. Переходные формы микрокапель концентрированной фазы, возникающие при последовательном увеличении напряженности вращающегося магнитного поля (частота поля $500 \mathrm{~Hz}$, напряженность поля слева направо и сверху вниз: 0.1, $0.2,0.5,1 \mathrm{kA} / \mathrm{m})$.
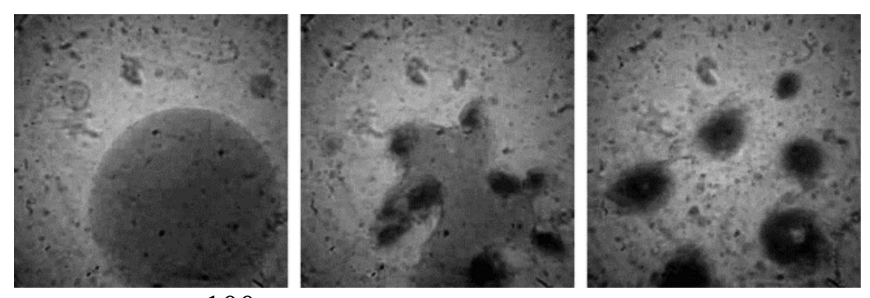

$100 \mu \mathrm{m}$

Рис. 4. Распад микрокапель концентрированной фазы во вращающемся магнитном поле (частота поля $40 \mathrm{~Hz}$, напряженность $1 \mathrm{kA} / \mathrm{m}$ ) при последовательном увеличении дополнительного перпендикулярного стационарного магнитного поля (напряженность стационарного поля слева направо: $0,0.5$, $1 \mathrm{kA} / \mathrm{m})$.

нескольких микрокапель их магнитное взаимодействие практически сразу приводит к слиянию микрокапель в одну более крупную, вследствие чего эффекты их совместного движения в поле, подобные описанным в работе [20], в данном случае не наблюдаются.

Проводились также исследования поведения микрокапель при одновременном воздействии вращающегося и стационарного магнитных полей. Вначале включалось однородное вращающееся магнитное поле, при этом капли принимали форму сплюснутых в плоскости вращения поля дисков. Затем дополнительно (квазистационарно) подавалось вертикальное магнитное поле (перпендику- лярное плоскости вращения). При этом на поверхности микрокапли возникали волнообразные возмущения (рис. 4). При дальнейшем росте напряженности вертикального поля эти возмущения трансформировались в крупные гребни, перемещающиеся вдоль поверхности капли, рост которых затем приводил к распаду капли на множество мелких вращающихся капель.

\section{Динамика макроскопической капли магнитной жидкости, содержащей микрокапли концентрированной фазы}

Для изучения динамики макроскопической капли магнитной жидкости, содержащей микрокапли концентрированной фазы, она помещалась на поверхность воды, принимая при этом сплюснутую форму, и вся система располагалась во вращающемся в горизонтальной плоскости магнитном поле. Видимый диаметр капли составлял $3 \mathrm{~mm}$. Измерялась частота вращения поверхности макроскопической капли в зависимости от частоты и напряженности внешнего магнитного поля. Напряженность магнитного поля при этом была такова, что не вызывала изменений формы капли, и вращение капли осуществлялось за счет внутренних вращений содержащихся в ней микрокапель концентрированной фазы. Для определения частоты вращения поверхности капли на нее наносилось несколько крупинок алюминиевой пудры. На рис. 5 представлена экспериментальная зависимость частоты вращения поверхности капли от частоты магнитного поля. Как видно из рисунка, при

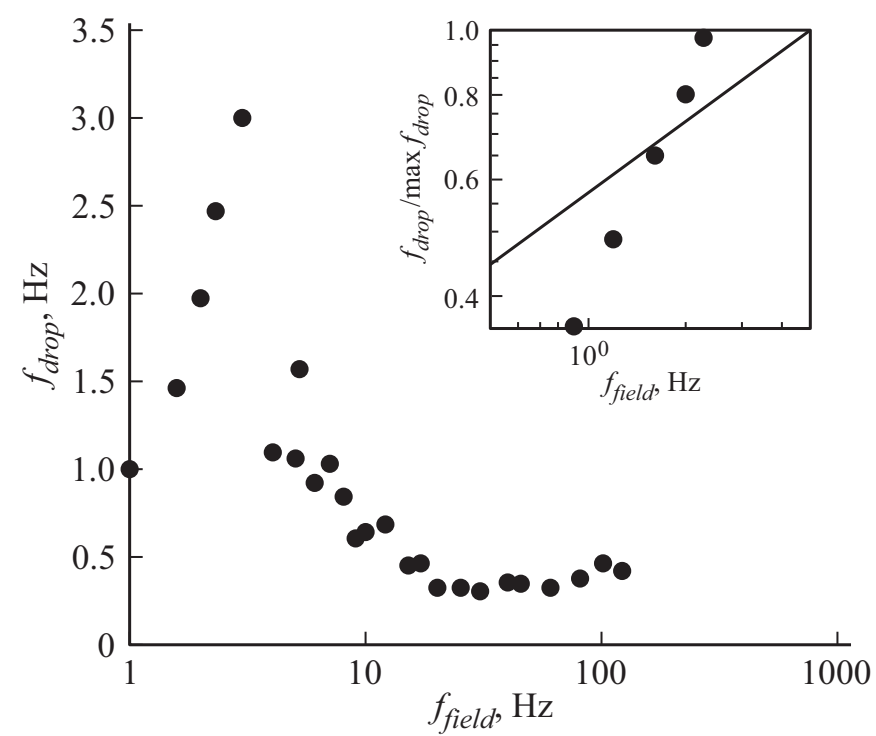

Рис. 5. Экспериментальная зависимость частоты вращения поверхности капли, содержащей микрокапли концентрированной фазы, от частоты вращения магнитного поля напряженностью $H=70 \mathrm{~A} / \mathrm{m}$. Во вставке показано сопоставление начальных участков экспериментальных и расчетных кривых, нормированных на максимальное значение частоты вращения капли. 


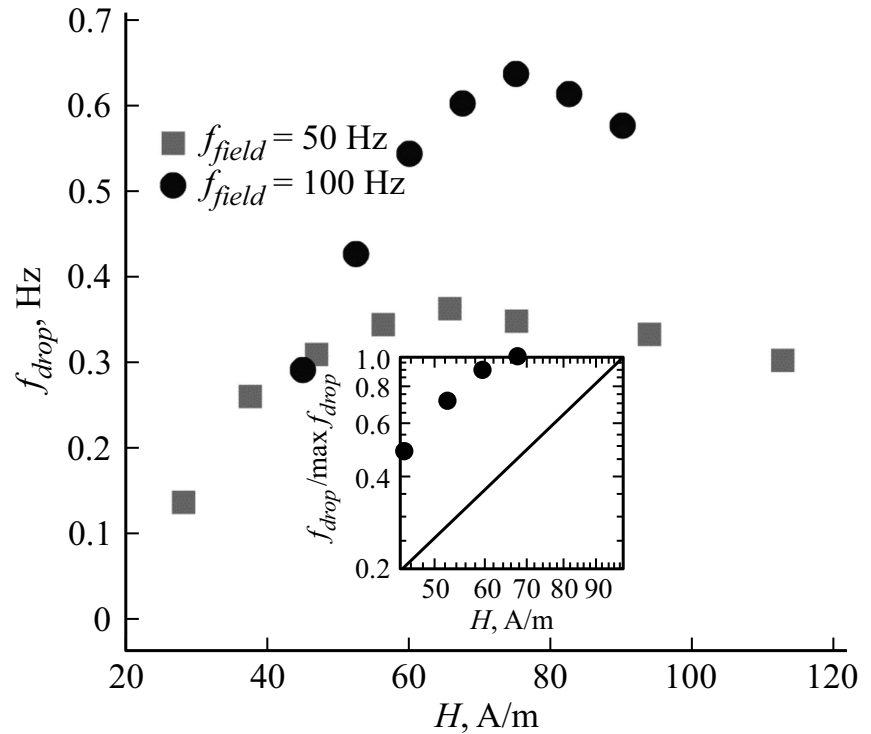

Рис. 6. Экспериментальные зависимости частоты вращения поверхности капли, содержащей микрокапли концентрированной фазы, от напряженности магнитного поля при различных значениях частоты вращения поля. Во вставке показано сопоставление начальных участков экспериментальных и расчетных кривых, нормированных на максимальное значение частоты вращения капли.

увеличении частоты вращающегося магнитного поля частота вращения капли вначале также возрастает, однако при достижении некоторого значения частоты поля, частота капли начинает уменьшаться. Такое поведение может быть связано с тем, что микрокапли концентрированной фазы в магнитной жидкости вначале (при низких частотах) имеют вытянутую вдоль поля форму, а затем с увеличением частоты поля трансформируются в переходные и дискообразные конфигурации, в результате чего передаваемый ими вращающий момент уменьшается.

На рис. 6 представлены экспериментальные зависимости частоты вращения поверхности макроскопической капли магнитной жидкости от напряженности магнитного поля. Как видно, данные зависимости носят немонотонный характер, что также можно связать с перестройкой геометрии микрокапель при увеличении поля.

\section{Обсуждение результатов и заключение}

Рассмотрим упрощенный механизм вращения капли магнитной жидкости, содержащей микрокапли концентрированной фазы. Если считать, что микрокапли не взаимодействуют и вращаются независимо, то их вклады суммируются пропорционально концентрации микрокапель [21]. Для частоты сферической капли магнитной жидкости, погруженной в немагнитную жидкость, имеем: $f_{0}=\eta_{1} / \eta_{2} f_{1} \varphi$, где $f_{1}$ - частота отдельной микрокапли, $\eta_{1}-$ вязкость внешней (немагнитной) жидкости, $\eta_{2}$ - вязкость среды, окружающей микрокапли. Частоту отдельной микрокапли можно оценить по формуле [11]

$$
f_{1}=\frac{\mu_{0}\left\langle H^{2}\right\rangle\left(2 \pi f_{\text {field }} \tau_{B}\right)^{1-\alpha}}{2 \pi\left(1+\chi n_{D}\right)^{2}} \chi \frac{n_{D}}{2 \eta_{2}}
$$

где $f_{\text {field }}$ - частота магнитного поля, $H$ - напряженность магнитного поля, $\tau_{B}-$ время релаксации намагниченности, $\chi$ - магнитная восприимчивость вещества микрокапли, $n_{D}-$ размагничивающий фактор формы капли, $\alpha=0.65$. Как видно, с увеличением частоты и напряженности вращающегося магнитного поля частота вращения капли магнитной жидкости, содержащей микрокапли концентрированной фазы, должна возрастать, что и наблюдается на начальных участках экспериментальных зависимостей (рис. 5, 6). Отметим, что экспериментальные данные и представленные расчеты в количественном отношении различаются весьма существенно (рис. 5, 6), что связано с качественным и весьма приближенным характером последних.

Данные рассуждения также не учитывают перестройку геометрии микрокапель и сложный характер динамики их формы, описанный в первой части настоящей работы. Именно с этими процессами должен быть связан наблюдающийся немонотонный характер зависимостей на рис. 5 и 6. Детальный анализ данных процессов представляет определенные трудности и выходит за рамки настоящей работы.

Таким образом, в настоящей работе была исследована новая композиционная среда на основе магнитного коллоида, представляющая собой суспензию микрокапель, имеющих высокую концентрацию магнитного вещества и способных реагировать на весьма слабые внешние магнитные поля. Исследована динамика формы таких микрокапель в стационарном и вращающемся магнитных полях. Выявлены возможные конфигурации формы, которыми могут обладать данные микрокапли. Показано возникновение макроскопического вращающего момента, действующующего на макроскопическую каплю магнитной жидкости, содержащую микрокапли концентрированной фазы, во вращающемся магнитном поле. Измерена зависимость частоты макроскопического вращения поверхности капли от частоты и напряженности магнитного поля. Полученные результаты могут представлять интерес в контексте решения проблем контроля и управления малыми объемами жидкого вещества при помощи внешних силовых полей.

Работа выполнена при поддержке Министерства образования и науки РФ в рамках базовой части государственного задания (проект № 3.5822.2017/8.9), а также при поддержке РФФИ (проект № 18-03-00279-а). 


\section{Список литературы}

[1] Afkhami S., Tyler A.J., Renardy Y., Renardy M., St Pierre T.G., Woodward R.C., Riffle J.S. // J. Fluid. Mech. 2010. Vol. 663. P. $358-384$.

[2] Lebedev A.V., Engel A., Morozov K.I., Bauke H. // New J. Phys. 2003. Vol. 5. P. 57.1-57.20.

[3] Drozdova V.I., Skibin Yu.N., Chekanov V.V. // Magnetohydrodynamics. 1981. Vol. 17. P. 320-324.

[4] Nguyen N.T., Beyzavi A., Ng K.M., Huang X. // Microfluid. Nanofluid. 2007. Vol. 3. P. 571-579.

[5] Zakinyan A., Nechaeva O., Dikansky Yu. // Exp. Therm. Fluid. Sci. 2012. Vol. 39. P. 265- 268.

[6] Nguyen N.T. // Microfluid. Nanofluid. 2012. Vol. 12. P. 1-16.

[7] Zakinyan A., Tkacheva E., Dikansky Y. // J. Electrostat. 2012. Vol. 70. P. 225-232.

[8] Zakinyan A., Beketova E., Dikansky Y. // Microfluid. Nanofluid. 2017. Vol. 21. Article ID: 88. doi: 10.1007/s10404-017-1924-5

[9] Диканский Ю.И., Закинян А.Р. // ЖТФ. 2010. Т. 80. Вып. 8. C. 8-12. [Dikansky Yu.I., Zakinyan A.R. // Tech. Phys. 2010. Vol. 55. P. 1082-1086.]

[10] Bacri J.C., Salin D. // J. Phys. Lett. 1982. Vol. 43. P. L-179-L-184.

[11] Bacri J.C., Cebers A., Perzynski R. // Phys. Rev. Lett. 1994. Vol. 72. P. 2705-2708.

[12] Janiaud E., Elias F., Bacri J.C., Cabuil V., Perzynski R. // Magnetohydrodynamics. 2000. Vol. 36. P. 301-314.

[13] Sandre O., Browaeys J., Perzynski R., Bacri J.C., Cabuil V., Rosensweig R.E. // Phys. Rev. E. 1999. Vol. 59. P. 1736-1746.

[14] Lebedev A.V., Pshenichnikov A.F. // Magnetohydrodynamics. 1991. Vol. 27. P. 4-8.

[15] Chaves A., Zahn M., Rinaldi C. // Phys. Fluid. 2008. Vol. 20. P. 053102.

[16] Torres-Diaz I., Cortes A., Cedeño-Mattei Y., Perales-Perez O., Rinaldi C. // Phys. Fluid. 2014. Vol. 26. P. 012004.

[17] Melle S., Calderón O.G., Fuller G.G., Rubio M.A. // J. Colloid Interf. Sci. 2002. Vol. 247. P. 200-209.

[18] Korobov M.I., Bedzhanyan M.A., Borisenko O.V., Dikansky Yu.I. // Exp. Therm. Fluid. Sci. 2017. Vol. 85. P. 69-74.

[19] Kolesnikova A., Zakinyan A. // Magnetohydrodynamics. 2018. Vol. 54. N 1-2. P. 45-48.

[20] Chen C.-Y., Hsueh H.-C., Wang S.-Y., Li Y.-H. // Microfluid. Nanofluid. 2015. Vol. 18. P. 795-806. doi: 10.1007/s10404-014-1472-1

[21] Pannacci N., Lemairea E., Lobry L. // Eur. Phys. J. E. 2009. Vol. 28. P. 411-417. doi: 10.1140/epje/i2008-10435-y 